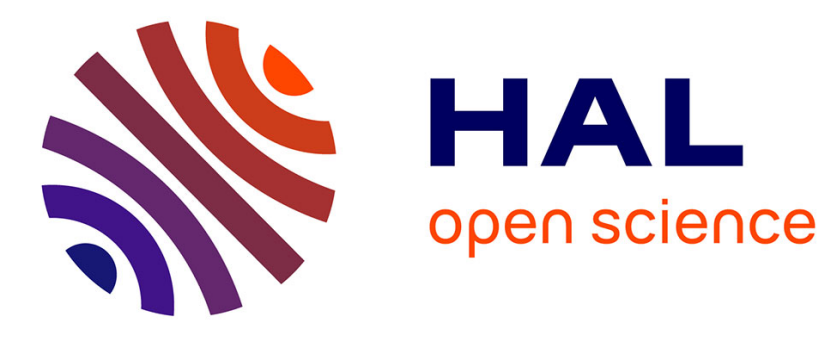

\title{
Generalised cyclidic nets for shape modelling in architecture
}

\author{
Romain Mesnil, Cyril Douthe, Olivier Baverel, Bruno Leger
}

\section{To cite this version:}

Romain Mesnil, Cyril Douthe, Olivier Baverel, Bruno Leger. Generalised cyclidic nets for shape modelling in architecture. International Journal of Architectural Computing, 2017, 15 (2), pp.148168. 10.1177/1478077117714917. hal-01520152

\section{HAL Id: hal-01520152 \\ https://hal-enpc.archives-ouvertes.fr/hal-01520152}

Submitted on 12 May 2017

HAL is a multi-disciplinary open access archive for the deposit and dissemination of scientific research documents, whether they are published or not. The documents may come from teaching and research institutions in France or abroad, or from public or private research centers.
L'archive ouverte pluridisciplinaire HAL, est destinée au dépôt et à la diffusion de documents scientifiques de niveau recherche, publiés ou non, émanant des établissements d'enseignement et de recherche français ou étrangers, des laboratoires publics ou privés.

$$
\text { Copyright }
$$




\title{
GENERALISED CYCLIDIC NETS FOR SHAPE MODELLING IN ARCHITECTURE
}

\author{
Romain MESNIL ${ }^{1,2}$, Cyril DOUTHE ${ }^{1}$, Olivier BAVEREL ${ }^{1}$, Bruno LEGER $^{2}$ \\ 1 NAVIER, UMR 8205, École des Ponts, IFSTTAR, CNRS, UPE, Champs-sur-Marne, France \\ ${ }^{2}$ Bouygues Construction, Guyancourt, FRANCE
}

\begin{abstract}
The aim of this paper is to introduce a bottom-up methodology for the modelling of free-form shapes in architecture that meet fabrication constraints. To this day, two frameworks are commonly used for surface modelling in architecture: NURBS modelling and mesh-based approaches. The authors propose an alternative framework called generalised cyclidic nets that automatically yields optimal geometrical properties for the envelope and the structural layout, like the covering with planar quadrilaterals or hexagons. This framework uses a base circular mesh and Dupin cyclides, which are natural objects of the geometry of circles in space, also known as Möbius geometry. This paper illustrates how complex curved shapes can be generated from generalised cyclidic nets. It addresses the extension of cyclidic nets to arbitrary topologies with the implementation of a 'hole-filling' strategy and demonstrates also that this framework gives a simple method to generate corrugated shells.
\end{abstract}

\section{Keywords:}

Free-form, conceptual design, structural morphology, architectural geometry, cyclidic net, fabrication-aware design, PQ-mesh, corrugated shell.

\section{INTRODUCTION}

The last three decades have seen the emergence of complexly shaped buildings, modelled with contemporary computer-aided design tools. Moving away from modernism, architects explore new formal possibilities offered by computers. Some spectacular realisations focused the attention of the media and have often associated so-called free-form architecture with the idea of limitless creativity, but also of high budget and delay. The materialisation of complex architectural shapes is subject to compromise between architectural intent and economical constraints, which are often related to fabrication and manufacturing of the components of the envelope or structure.

The morphogenesis of complex architectural shapes has been conceptualised by Motro [1] and later by Bagneris et al., who identify three design strategies for freeform structures [2].

- Geometrically-constrained morphogenesis considers shapes with properties that simplify their fabrication. This approach has been used for centuries: it is based on relatively simple shapes, like surfaces of revolution or developable surfaces. The feasibility of the materialisation is therefore at the cost of a reduced formal freedom. 
- Mechanical-constrained morphogenesis considers shapes that have good mechanical properties. Those shapes are typically the result of mechanical form-finding. This field was pioneered by Frei Otto and his team: textile architecture or funicular shells can be classified in this 'form follows force' approach.

- Flexible morphogenesis considers other aspects, like comfort, ornamentation. NURBS modelling falls into this category because it does not inform the designer on the constructability or structural performance of the shape he or she is drawing. Flexible morphogenesis requires post-rationalisation to meet structural or fabrication constraints. This makes the design process more complex, as the interplay between architects, geometry specialists and structural engineers [2].

The present paper proposes an extension of geometrically-constrained morphogenesis, where form is steered according to technological constraints. Fabrication constraints have indeed an important impact on the economy of a doubly curved envelope and dictate the choices of designers during construction. One of the most notable examples of recent improvements of geometrically-constrained morphogenesis approach are scale-trans surfaces, a family of shapes covered by planar quadrilaterals introduced by Glymph et al. [3]. The shapes generated in this paper consider following construction properties:

- The covering of surfaces with planar quadrilateral or hexagonal facets, which makes the use of costly moulds unnecessary and reduces the cost of manufacturing, especially for glazed envelopes.

- The offsets without geometric torsion of nodes, which simplifies the construction of the structural layout (see Figure 1 for a visual explanation) and allows for the construction of double-layer structures.

- The parameterization of surfaces by circular arcs, which opens possibilities for the construction of curved support structures with conventional technologies.

More specifically, and in the following of [4], this paper proposes the application of recent advances in the field of discrete differential geometry for fabrication-aware computer modelling and introduces generalised cyclidic nets, a new framework for fabrication-aware shape generation. The aim of this article is to detail the implementation of generalised cyclidic nets and to illustrate the potential of geometrically-constrained design approach for shape modelling in architecture. Main contributions include:

- a strategy for hole-filling with cyclidic nets, generalizing the idea of [5] for cyclidic nets of arbitrary topology;

- an extension of the notion of cyclidic nets to generalised cyclidic nets, which allows for a discontinuity of the normal vector and give new ways to model corrugated shells;

- and as a result, a new framework tailored for complex shape generation in architecture based on cyclidic nets.

The paper is organised as follows: section 2 introduces key geometrical notions used in the framework introduced by the authors. Section 3 proposes an extension of cyclidic nets to arbitrary topologies. The modelling framework for free-form architecture based on these findings is then presented in section 4. A brief conclusion sums up the findings of this paper and proposes some developments to this work. 


\section{CYCLIDIC NETS AND MÖBIUS GEOMETRY}

\subsection{Möbius Geometry}

We define first here some key concepts related to architectural design of curved shapes. We recall the link between continuous and discrete geometry, and especially the role of lines of curvatures for geometrical optimal design of quad meshes.

\section{Circular meshes}

The importance of using planar elements for cladding has been identified early in the history of free-form architecture. Triangular meshes provide planar elements, but quad meshes are often preferred because of simpler connection details. Due to their lower node valence, quad meshes also achieve a higher transparency than triangular meshes. This led to a research effort towards planar quads (PQ)-meshes $[1,6,7]$.

Architectural designs require a structural depth, and therefore, an offset of the reference geometry, generally defined as a mesh. The notion of torsion-free node was thus introduced to define meshes where planar beams resting on the mesh edges meet along a common axis at each node. Figure 1(a) represents a torsion-free node. Figure 1(b) displays a node, which is not torsion-free: notice that the nonalignment of the beam axes is accommodated by the size of the node.

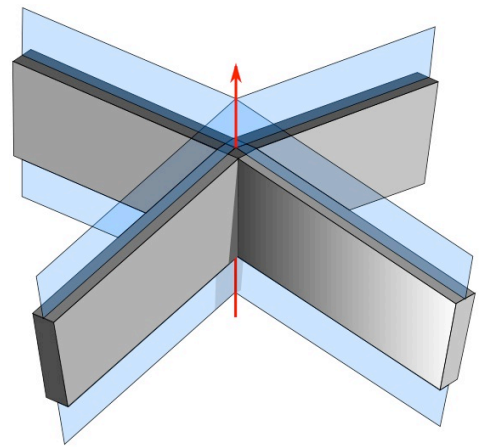

(a) A torsion-free node (inspired by [8])

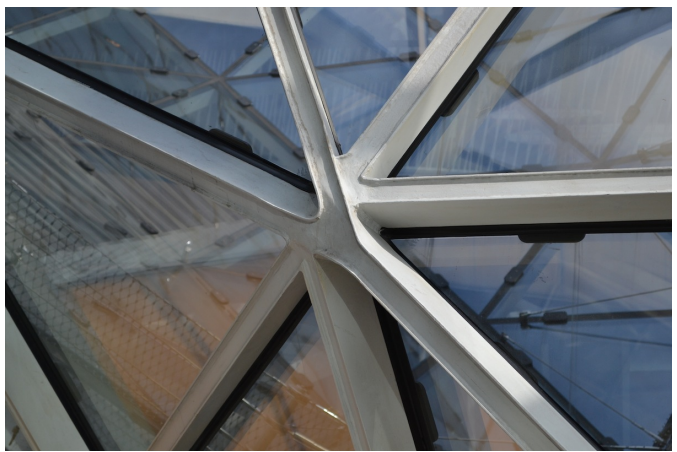

(b) A node with geometric torsion

(picture: Romain Mesnil)

Figure 1: Illustration of geometric torsion in gridshell structures.

It possible to generate simple torsion-free beam layouts by setting the node axes along a constant vector, but this solution is impractical for highly curved surfaces. Indeed, ideally, the node axes of a torsion-free beam layout should be normal to the surface. Only few families of meshes have this property, among them circular meshes, which constitute an important class of quadrilateral meshes where each face is inscribed in a circle. These meshes have two interesting characteristics for architecture. First they admit constant vertex offset and therefore a non-trivial torsion-free layout. Second they are seen as a discrete parameterization of surfaces by lines of curvature [7].

The notion of non-trivial mesh offset is actually closely linked to the lines of curvature of smooth surfaces. A surface parameterised by its lines of curvatures yields automatically meshes that are close to circular meshes $[7,9,10]$. It has also been noticed that planar hexagonal meshes are closely related to Dupin indicatrices of smooth surfaces, and that the optimal placement of hexagonal cells follows lines of curvature [11]. An example of such a mesh is shown in Figure 2. The hexagonal layout has been generated following lines of curvatures of the surface, the optimisation towards planarity being performed using dynamic relaxation [12]. 


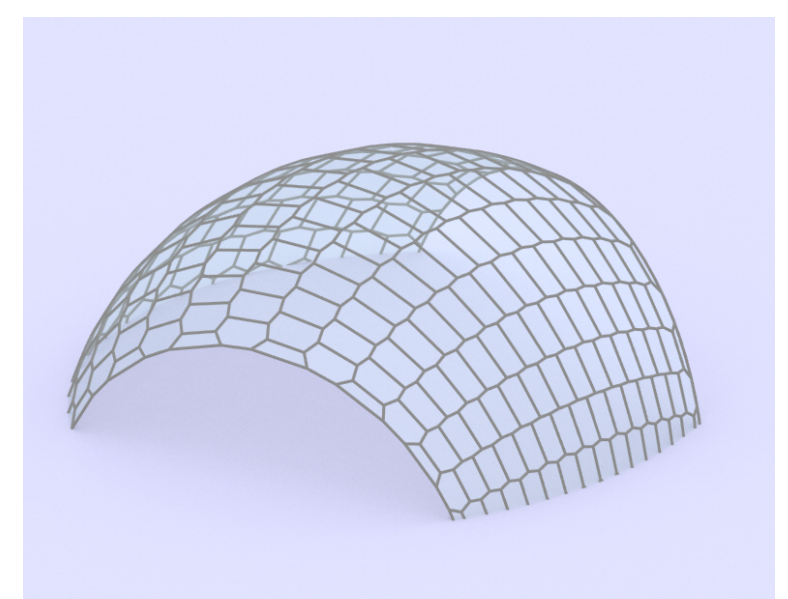

Figure 2: A surface covered with planar hexagonal panels following lines of curvature.

\section{Möbius transformations and Möbius geometry}

Lines of curvature play thus a crucial role in the discretisation of complex shapes. It is legitimate to study transformations that preserve lines of curvature. A particular group of such transformations is known as Möbius transformations, which are combinations of spherical inversions and homothetic transformations. Möbius transformations also map circles to circles, and therefore map circular meshes to circular meshes. This study will focus on discrete surfaces where all faces are inscribed within circles [13], and thereby surfaces naturally meshed by their lines of curvature. So, the general framework of this study is the geometry of circles in space, also called Möbius Geometry. To go into detail, a spherical inversion is defined by a centre $\mathbf{C}$, and a ratio $k$. The image $\mathrm{M}^{\prime}$ of a point $\mathrm{M}$ by a spherical inversion is given by Equation (1). A spherical inversion leaves the sphere of centre $\mathbf{C}$ and radius $\sqrt{|k|}$ unchanged.

$$
\left\{\boldsymbol{C}, \boldsymbol{M}, \boldsymbol{M}^{\prime}\right. \text { aligned }
$$

It is interesting to notice that spherical inversions do not preserve the global shape, except spheres. These transformations give therefore a way to apply global deformations to meshes while preserving local properties. The potential of these simple transformations is illustrated in section 4.

\subsection{Cyclidic nets}

\section{Dupin cyclides}

This work uses a key object in discrete differential geometry: Dupin cyclides. These surfaces were discovered by the French mathematician Charles Dupin, who studied some of their remarkable properties in 1803. Dupin cyclides can be defined as inversion of tori in the sense of Section 2.1. Some special cases of Dupin cyclides include tori, cylinders and spheres. An example of a cyclide with patches following the lines of curvature is shown in Figure 3. 

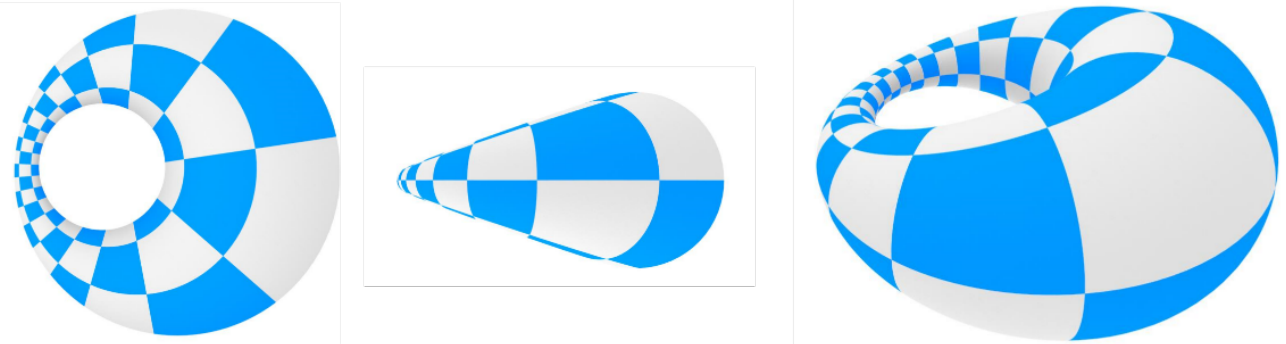

Figure 3: Dupin's cyclide parameterised by its lines of curvature (in order top, front and perspective views).

For application in architecture, some properties of cyclides are particularly appealing:

- their lines of curvature are circles;

- a quad whose edges are lines of curvature is inscribed in a circle: lines of curvature thus naturally create circular meshes;

- they are isothermic surfaces [14] and can therefore be covered with Edge Offset Meshes [15].

Beside, Dupin cyclides are easily parameterised by lines of curvature. Indeed, think of them as spherical inversions of tori: the image of the lines of curvature of a torus (the parallels and meridians) by spherical inversions remain lines of curvature of the resulting Dupin cyclide. This guarantees good properties for other meshes than quad meshes, like hexagonal meshes [11] and possibly for other patterns, like tri-hex meshes [16].

\section{Cyclidic patch}

The four intersections of four lines of curvature in cyclides naturally define a circle. Conversely, a cyclic quadrilateral and a frame give a unique portion of cyclide, later called cyclidic patch in this paper. Several algorithms have been proposed to convert a cyclidic patch to a NURBS surface. The one used in this paper has been proposed in [17]. The algorithm requires a cyclic quadrilateral and an arbitrary orthogonal frame in 3D (one blue and one red arrow in Figure 4). The two vectors are tangent vectors to the edges of the resulting surface. The other frames are generated by reflection with respect to the median plane of each edge of the quad and define the next tangent vectors of the borders of the cyclidic patch. Notice how the median planes of each edge intersect at the centre of the circumcircle of the quadrangle in Figure 4: this only occur for circular quadrilaterals and illustrates why they are required as input for cyclidic patch. The boundaries of the patch are circles which are uniquely defined by two points and an orthogonal frame defining tangent vectors of the patches.
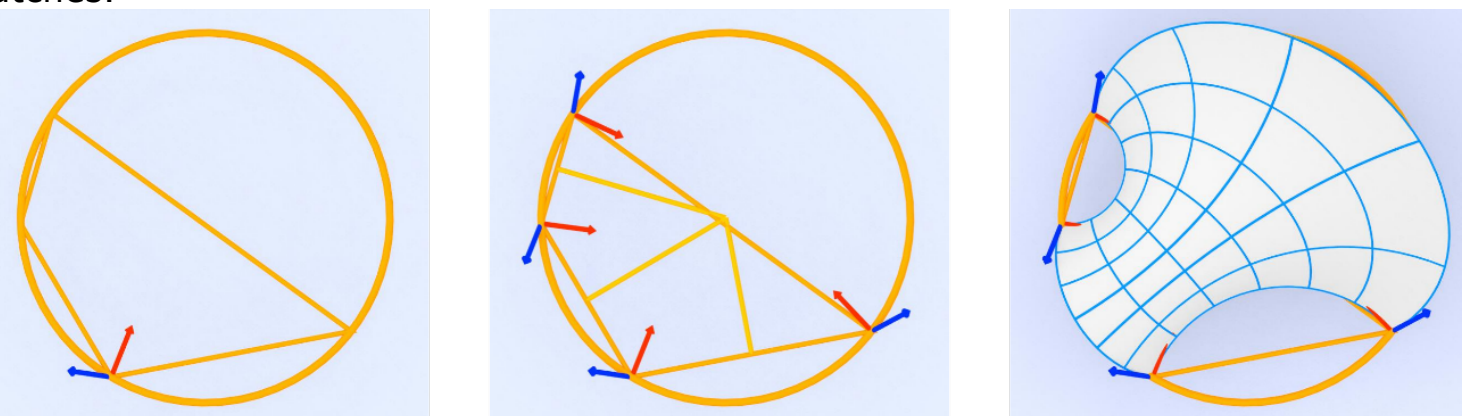

Figure 4: Cyclidic patch: the four corners are on the same circle, the surface is naturally parameterised by its lines of curvature. The frames define tangent vectors for the edges of the patch. 
The algorithm proposed in [17] is designed so that the isoparametric curves of the resulting patch are lines of curvature. This is possible because analytical expression on the parameterization of Dupin cyclides by their lines of curvature exist. The resulting surface is thus naturally parameterised by its lines of curvatures. This means that the trivial quad meshes on cyclidic patches like the one displayed in Figure 4 or Figure 5 are exact circular meshes.
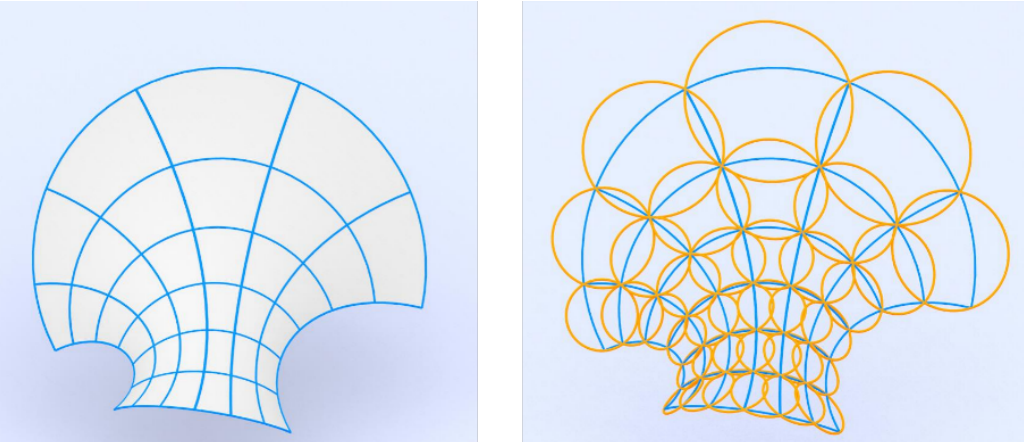

Figure 5: Left: Cyclidic patch meshed along its lines of curvature. Right: the associated circumcircles.

\section{Cyclidic nets}

The properties of cyclides and the existence of a conversion algorithm to NURBS led to the idea of representing shapes as a collection of cyclidic patches. The mathematical properties of such shapes, called cyclidic nets, have been studied in [18]. Cyclidic nets are based on Circular Quadrilateral Meshes and require only one frame vector, the others being generated by reflection if they belong to the same cyclidic patch (see Eqn (2), in Section 3.1). A simple reflection rule illustrated in Figure 6 allows the propagation of the frame to adjacent patches.
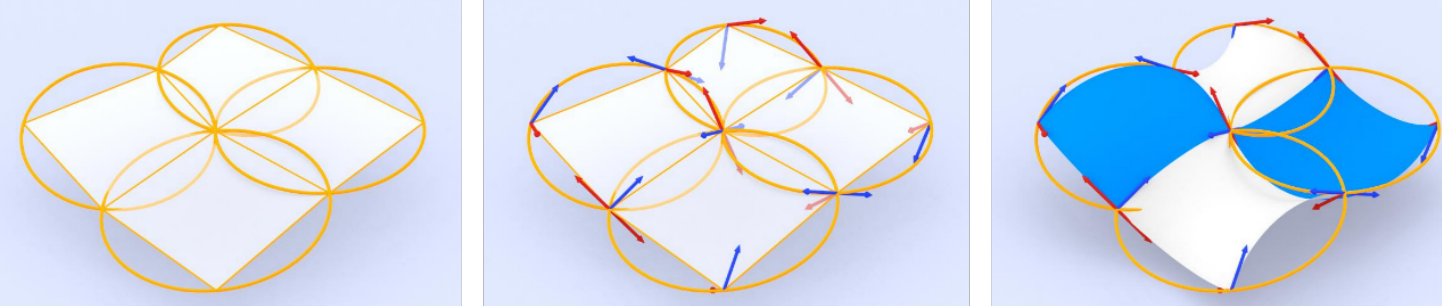

Figure 6: Left: a coarse circular mesh. Middle: the frames propagated by reflection.

Right: the resulting cyclidic net.

Applications of cyclidic nets for architecture were proposed in [19], the cyclidic patches being typically the size of panels. This solution has the advantage of generating one unique type of node, but it requires building with circular arcs. This solution would also require remeshing if the designer wants to modify the structural layout. However, considering the fact that a cyclide can easily be meshed by its lines of curvature to form a circular mesh, one might see cyclidic nets as a tool to define the global shape and the underlying discretisation of each cyclidic patch as the real structural layout. Doing so, the designer works with circular meshes, keeping the density of the mesh as a parameter almost independent of the shape. The opportunity offered by larger cyclidic patches as global modelling tool is unexplored up to now.

\subsection{Field of application of classical cyclidic nets}


The use of cyclidic nets in the field of computer-aided geometric design has been proposed as early as in the 1980's [20,21]. Some of their properties are particularly interesting: they have a rational offset and a low algebraic degree, meaning that geometrical operations can efficiently be performed on cyclidic nets. However, practical limitations -the main one being the difficulty to model surfaces with umbilical points- restricted their possible field of application. Indeed, Dupin's cyclides do not have umbilical points: their lines of curvature and therefore the edges of the cyclidic patches are thus strictly perpendicular. Consequently, smooth cyclidic nets require that exactly four patches meet along a common vertex, so that shapes with complex topologies cannot be modelled. To cope with this limitation, the next section develops three new strategies that extend the field of possible shapes and topologies for smooth and non-smooth cyclidic nets.

\section{EXTENDING CYCLIDIC NETS TO ARBITRARY TOPOLOGIES}

\subsection{Corrugated shells with generalised cyclidic nets}

Corrugated shells are of importance for architectural design, as creases, understood as discontinuity of the normal vector on a surface, are an element of architectural language, for example in Frank Gehry's architecture. A detailed review of folded structures is presented in [22] and illustrates the interest of designers to nonsmooth architectural shapes. Corrugated shells are also known to be of interest in structural engineering, because they locally increase Gaussian curvature and can thus improve the buckling strength of thin shells. French engineer Nicolas Esquillan used doubly curved creases for his record-breaking concrete shells [23] including the CNIT, which remains the longest spanning shell in the world to this day. In Esquillan's work, corrugated shells were however restricted to a limited family of shapes and have not revealed their full potential. A study carried out in [24] demonstrates that corrugation significantly improves the structural efficiency of thin shells for configuration with free-edges. This element of language is however in need of appropriate modelling tools, as current research on geometrically-constrained morphogenesis emphasizes on smooth shapes $[4,6,8]$. In this paper, the authors generalise the construction rules of cyclidic nets to deal with discontinuities of normal vectors.

The fundamental shapes remain cyclidic patches, the only difference with the usual cyclidic nets as defined in $[18,21]$ is the reflection rule for the normal vectors, as illustrated on the left of Figure 7.
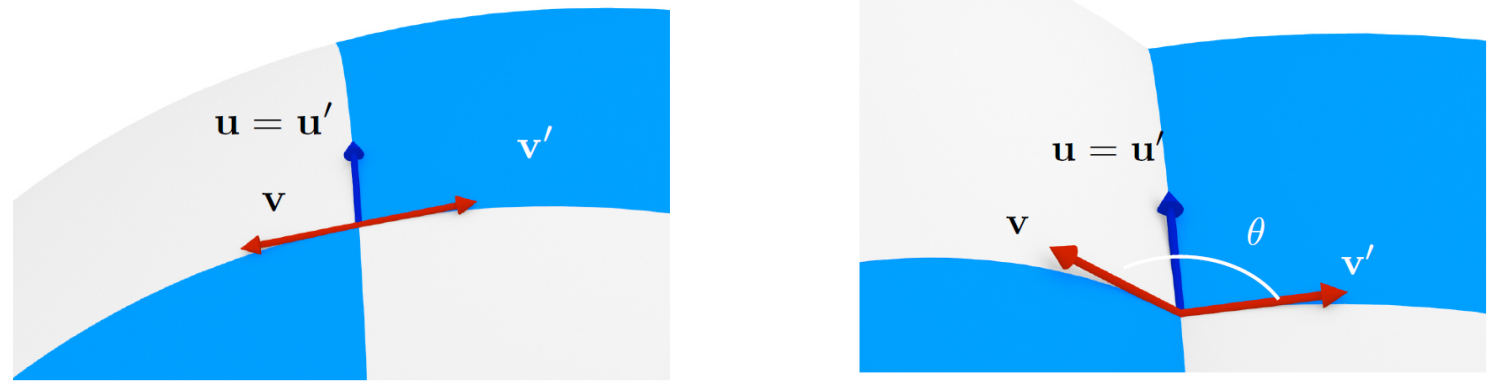

Figure 7: Left: reflection rule for classical cyclidic nets. Right: reflection rule for generalised cyclidic nets.

When propagating the frame of the cyclidic patch from one face to the other, the classical approach keeps one vector and inverts the other: 


$$
\left\{\begin{array}{c}
\boldsymbol{u}^{\prime}=\boldsymbol{u} \\
\boldsymbol{v}^{\prime}=-\boldsymbol{v}
\end{array}\right.
$$

In Equation (2), $\boldsymbol{u}$ refers to the common edge between the two patches. The first equality means that two patches have the same boundary; the second equality translates the continuity of tangent vectors to form a $\mathrm{C}^{1}$ surface. Therefore the second equality is not necessary if one only deals with $C^{0}$ surfaces. Equation (2) can thus be written in a more general form, illustrated in the left of Figure 7:

$$
\left\{\begin{array}{c}
u^{\prime}=u \\
v^{\prime}=R_{u, \theta} \cdot v
\end{array}\right.
$$

$\boldsymbol{R}_{\boldsymbol{u}, \boldsymbol{\theta}}$ is a matrix representing the rotation around the axis $\boldsymbol{u}$ by an angle $\theta$ called crease angle in this paper. Equation (2) is therefore a specific case of Equation (3), with an angle $\theta=\pi$. This generalised definition has to be consistant for any patch, which implies that the crease angle has to be constant along a polyline. Equation 3 can be propagated because lines of curvature have a zero geodesic torsion: the angle made by the normal of a line of curvature and the surface normal is constant. Another restriction is that it is not possible to introduce creases in two different directions. The continuity of the surface would be lost otherwise. A few examples of creased structures are shown in section 4.

\subsection{Closed nets}

A significant issue linked with the practical modelling with cyclidic nets is the construction of closed nets forming loops. Indeed, a closed circular mesh does not necessarily yield valid cyclidic nets, as illustrated in Figure 8 . In this figure, the reflection rule is applied along the closed strip, and the resulting surface is not continuous.

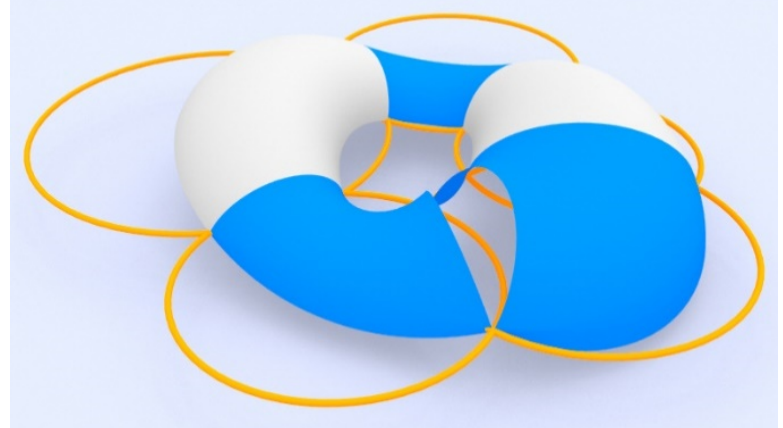

Figure 8 : A closed circular mesh which yields invalid cyclidic nets

For the sake of simplicity, consider a unique strip constituted of $N$ circular quads. A cyclidic net can be generated by propagating an orthogonal frame chosen arbitrarily on the first quad. Consider the frame vectors $\boldsymbol{u}_{i}$ and $\boldsymbol{v}_{i}$ that define the boundaries of cyclidic patches already depicted in Figure 4 and 6 , and their propagation along a closed polyline. They are defined by:

$$
\forall i>0, \boldsymbol{u}_{i}=T_{i-1} \circ T_{i-2} \circ \ldots \circ T_{0}\left(\boldsymbol{u}_{0}\right)
$$

where $T_{i}$ is the reflection with respect to the median plane of the $\mathrm{i}^{\text {th }}$ edge. A closed smooth cyclidic net should reconnect itself with a continuity of tangent vectors, and therefore should verify the following equation:

$$
T_{N} \circ T_{N-1} \circ \ldots \circ T_{0}\left(\boldsymbol{u}_{0}\right)=\boldsymbol{u}_{0}
$$


Ideally, the designer would like to select any $\boldsymbol{u}_{0}$ arbitrary and that it verifies Eqn (5). Mathematically, this problem is exactly the same as the existence of constant vertexoffset for circular meshes, a topic covered in [25]. Constant vertex-offset of quadrilateral meshes is linked with the existence of a mesh with its edges parallels to the ones of a given mesh, and whose vertices are inscribed within a sphere. The normal vector chosen at each vertices correspond then to the vector pointing from the centre of the sphere to the vertices. Equation 5 is already studied in [25], and following statements can thus be made based on their work:

- closed continuous cyclidic nets are supported on circular meshes admitting a constant-vertex offset;

- this is equivalent to the fact that the boundary has to be quasi-spherical (i.e. there exists a parallel curve which has its vertices inscribed in a sphere);

- the border of a continuous cyclidic net can be filled with a continuous net, since it is a pseudo-spherical curve.

The second proposition can be simplified in the case of a planar curve discussed in this paper. The closing condition is equivalent to the fact that the boundary curve is parallel to a curve inscribed in a circle. This is expressed simply with an angular criterion used within the proposed framework. For an even-sided planar curve, we introduce $(\alpha)$ the external angles of the curve, shown in Figure 9 and have:

$$
\sum_{k=0}^{N}(-1)^{k} \alpha_{k}=0
$$

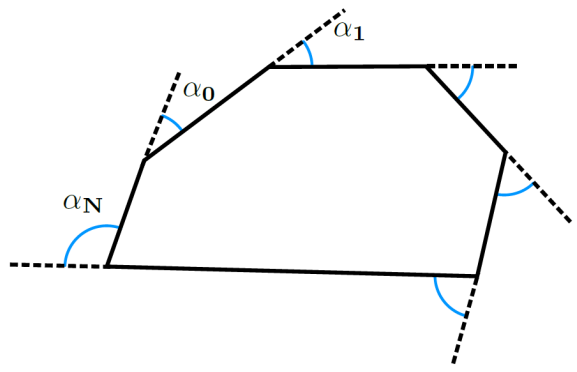

Figure 9: A closed planar curve and its external angles

The composition rule for reflections applies if the transition between two cyclidic patches is governed by equation (2). Since the first equalities of Equations (2) and (3) are identical, the reflection described in Equation (4) are unchanged. It follows that the statements made on cyclidic nets can be extrapolated to generalised cyclidic nets.

\subsection{Hole-filling problem}

\section{Principle of the algorithm}

The main limitation with cyclidic nets as a design tool is the design of complex topologies. The fact that two consecutives edges on a cyclidic patch have to be perpendicular makes it impossible to assemble more than four patches around a vertex. This difficulty has already been pointed out in [5] and [19]. In [19], the authors propose to leave openings in cyclidic nets and fill them with a spherical cap. This requires an optimisation of the basis circular mesh and of the orientation of the frame used to generate the cyclidic net. Moreover, this strategy works for openings with two arcs. In [5], a hole-filling strategy has been proposed for openings with more than four edges. This method only works for symmetrical shapes and can therefore not be applied in a general framework. A general method extending this proposition and allowing for non-symmetrical hole filling is thus proposed here. 
From a practical point of view, the designer leaves an opening in a cyclidic net. The opening is bounded by edges of cyclidic patches, which are circular arcs. Because we aim at modelling complex topologies, the hole has more than four edges. We do not impose any restriction on the geometrical configuration of the edges of the opening, besides the fact that consecutive edges should be perpendicular. The aim of the hole filling strategy is to shrink the opening down to a hole of the size of a panel. This requires an iterative procedure: in the example of Figure 10, the filling strategy has been applied three times. For a $n$-sided hole, the proposed method leaves $n$ parameters for the designer for each step of a hole filling strategy, which makes it difficult to control the overall smoothness of the surface. It is therefore necessary to automate the choice of these variables. A simple way to do this is to minimise a smoothness measurement. An example of target function is the distance of the new corner points to a circle. The choice of other functions is discussed in Section 3.4.

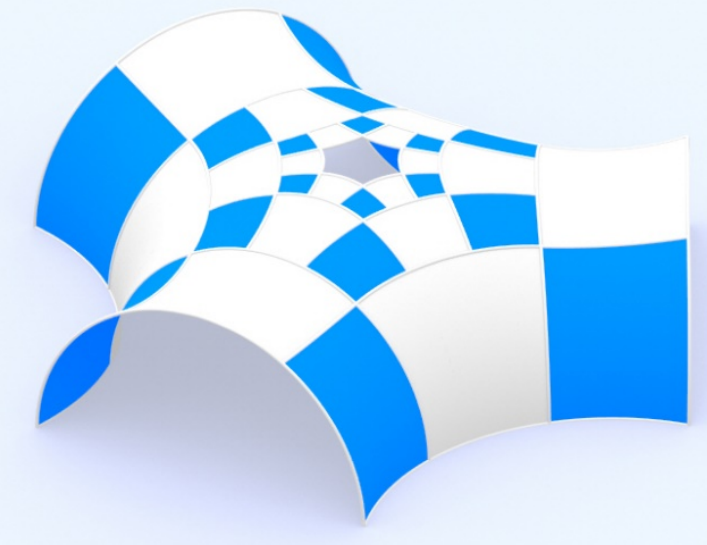

Figure 10: The result of the hole filling strategy, with smooth T-joints between patches.

\section{Krasauskas' algorithm}

Consider Figure 11, the hole to fill has six circular edges. We write $\boldsymbol{V}_{\boldsymbol{i}}$ the vertices and $C_{i}$ the circular edge going from $\boldsymbol{V}_{\boldsymbol{i}}$ to $\boldsymbol{V}_{\boldsymbol{i}+\mathbf{1}}$. The designer must chose two points $\boldsymbol{P}_{\boldsymbol{i}}^{\boldsymbol{L}}$ and $\boldsymbol{P}_{i}^{R}$ on each circular $\operatorname{arc} C_{i}$. The algorithm proposed in [5] is:

1. Choose arbitrarily the $P_{i}^{L}$;

2. Find the $\boldsymbol{P}_{i}^{R}$ so that $\boldsymbol{P}_{i}^{L}, \boldsymbol{P}_{i+2}^{R}$ and $C_{i+1}$ belong to the same sphere. At this step all the points on the boundaries are chosen.

3. Draw the corner circles passing through $\boldsymbol{V}_{\boldsymbol{i}} \boldsymbol{P}_{\boldsymbol{i}}^{L} \boldsymbol{P}_{i}^{R}$, defined in Figure 11;

4. Choose any point $\boldsymbol{P}_{\mathbf{0}}$ on the first circle and create the middle circles by propagation.

The main difficulty with this method is that the last quadrangle, highlighted in red on Figure $11 \mathrm{~d}$ is a priori not inscribed within a circle, unless some symmetries are at stake. The system is thus over-constrained. We prove here that one additional condition has to be imposed on one of the $\boldsymbol{P}_{\boldsymbol{i}}^{\boldsymbol{L}}$ in order to yield exact circular meshes supported on the boundary arcs, and thus valid cyclidic nets. 


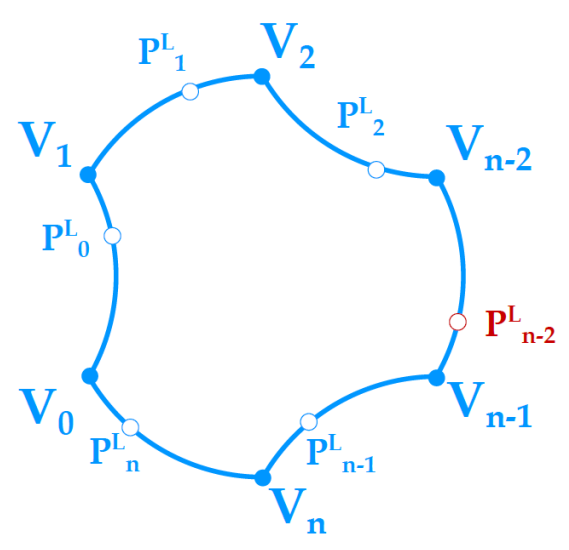

(a) Choice of the $P_{i}^{L}$

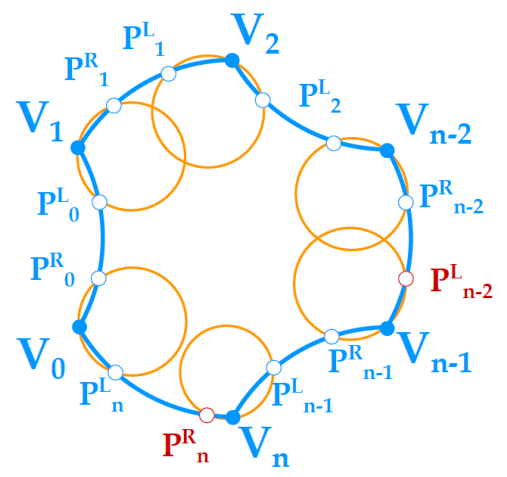

(c) Construction of the corner circles

Figure 11: The steps of the hole-filling strategy, the Propagation of a circular mesh order to have a closed circular strip.

\section{Preliminary statements}

We suppose that all the $\boldsymbol{P}_{i}^{L}$ but one, for example $\boldsymbol{P}_{n-2}^{L}$, are chosen arbitrarily. The corresponding $\boldsymbol{P}_{i}^{R}$ are chosen with the rule explained hereinabove. We also suppose known the point $\boldsymbol{P}_{\mathbf{0}}$. This section shows how to determine a value of $\boldsymbol{P}_{n}^{\boldsymbol{R}}$ (equivalently $\boldsymbol{P}_{n-2}^{L}$ ) so that the quadrangle $\boldsymbol{P}_{n}^{L} \boldsymbol{P}_{0} \boldsymbol{P}_{n} \boldsymbol{P}_{n}^{R}$ depicted in Figure 12 is circular.

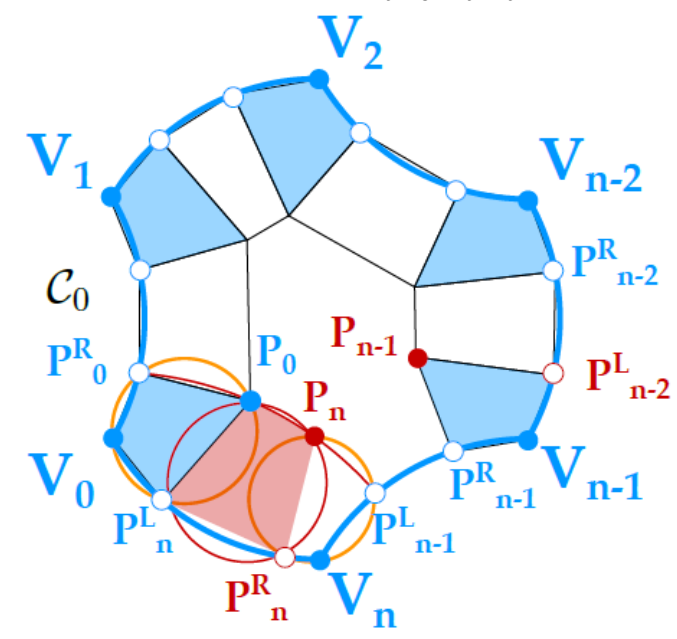

Figure 12: Notations of the hole-filling problem

Pre-print submitted to The International Journal of Architectural Computing (2017) 
We call $S_{n}$ the sphere defined by the points $\left(\boldsymbol{V}_{\mathbf{0}}, \boldsymbol{P}_{\boldsymbol{n}}^{\boldsymbol{L}}, \boldsymbol{V}_{\boldsymbol{n}}, \boldsymbol{P}_{\boldsymbol{n}-\mathbf{1}}^{\boldsymbol{L}}\right)$. We call $\boldsymbol{P}_{\boldsymbol{n}}$ the intersection of the circles $\left(\boldsymbol{P}_{n}^{L}, \boldsymbol{P}_{0}, \boldsymbol{P}_{n}^{R}\right)$ and $\left(\boldsymbol{P}_{n}^{R}, \boldsymbol{V}_{n}, \boldsymbol{P}_{n-1}^{L}\right)$. We call $\boldsymbol{P}_{n}^{\prime}$ the intersection of the circles $\left(\boldsymbol{P}_{n-1}, \boldsymbol{P}_{n-1}^{R}, \boldsymbol{P}_{n-1}^{L}\right)$ and $\left(\boldsymbol{P}_{n}^{R}, V_{n}, P_{n-1}^{L}\right)$.

The mesh generated is valid if and only if $\boldsymbol{P}_{\boldsymbol{n}}^{\prime}$ and $\boldsymbol{P}_{\boldsymbol{n}}$ are identical. We thus study more precisely the envelopes of this points when varying the position of $\boldsymbol{P}_{\boldsymbol{n}}^{\boldsymbol{R}}$ (equivalently $\boldsymbol{P}_{n-2}^{L}$ ). Three statements can be made:

1. The envelope of all the possible $\boldsymbol{P}_{\boldsymbol{n}}$ and $\boldsymbol{P}_{n}^{\prime}$ are in $S_{n}$;

2. The envelopes of all the possible $\boldsymbol{P}_{n}$ is a circle going through $\boldsymbol{P}_{\mathbf{0}}$ and $\boldsymbol{P}_{n-1}^{L}$;

3. The envelopes of all the possible $\boldsymbol{P}_{n}^{\prime}$ is a circle going through $\boldsymbol{P}_{\boldsymbol{n}-\mathbf{1}}^{L}$.

In other terms, the envelopes of $\boldsymbol{P}_{\boldsymbol{n}}$ and $\boldsymbol{P}_{n}^{\prime}$ are two circles belonging to the same sphere and having one point in common. Therefore, they have two points in common in general, the second one is the point $\boldsymbol{P}_{\boldsymbol{n}}=\boldsymbol{P}_{n}{ }_{n}$ we are looking for. Let us now look at the justification of these statements.

The first statement is not difficult to prove: by construction $\boldsymbol{P}_{n}^{R}, \boldsymbol{V}_{n}$ and $\boldsymbol{P}_{n-1}^{L}$ belong to $S_{n}$. Therefore, the circle going through these points belong to $S_{n}$.

The other two statements are less immediate. Consider the envelope of the $\boldsymbol{P}_{\boldsymbol{n}}$ : we can do an inversion with respect to any point of the circle $C_{n}$. The problem becomes then planar and is depicted on the left of Figure 13. Notations for angles are introduced on the right of Figure 13. The subscript $\boldsymbol{X}_{\boldsymbol{i}}$ indicates the image of $\boldsymbol{X}$ by the inversion.
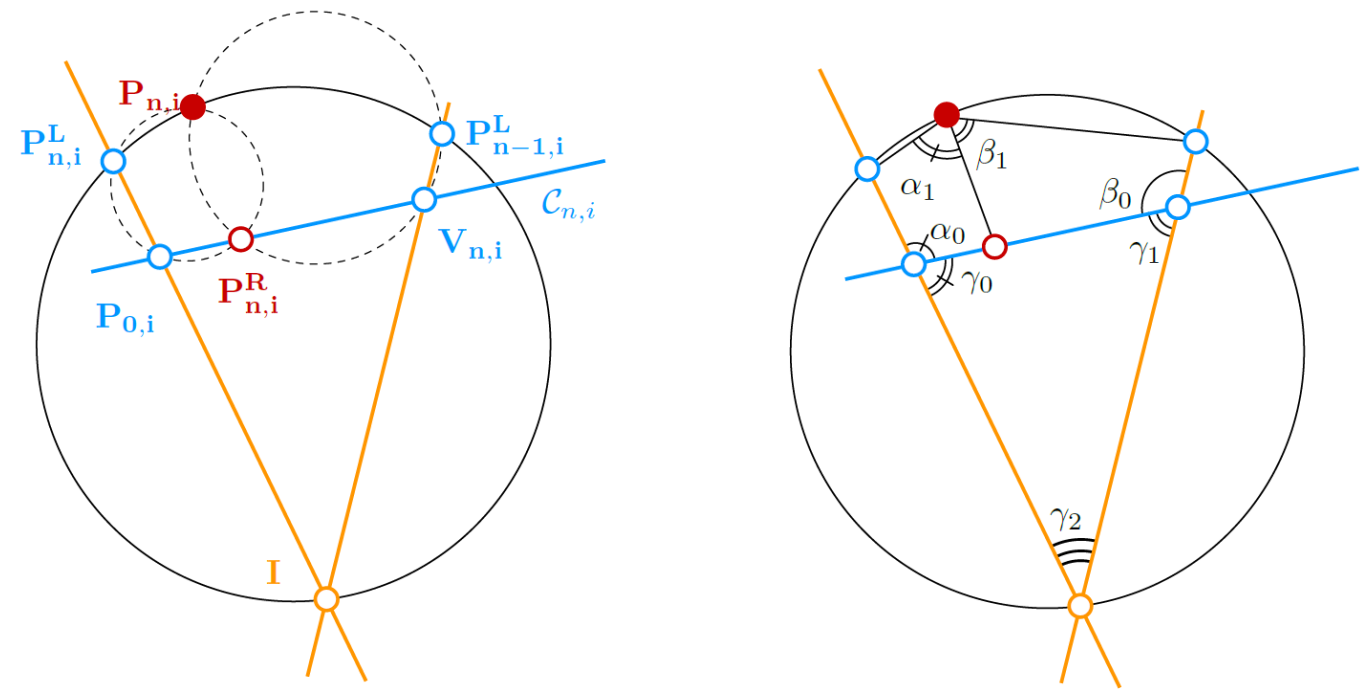

Figure 13: Left: the trajectory of all possible $P_{n}$ after inversion. Right: Notations used for angles

The image of $C_{n}$ is a straight line. We introduce the point $I$, intersection of the lines $\left(P_{0, i} P_{n, i}^{l}\right)$ and $\left(V_{n-1, i} P_{n-1, i}^{l}\right)$. By construction, we thus have:

$$
\left\{\begin{array}{c}
\gamma_{0}=\pi-\alpha_{0} \\
\gamma_{1}=\pi-\beta_{0} \\
\gamma_{0}+\gamma_{1}=\pi-\gamma_{2}
\end{array}\right.
$$


Recall that in circular quadrilaterals, the sum of opposite angles is equal to $\pi$. This yields:

$$
\left\{\begin{array}{l}
\alpha_{1}=\pi-\alpha_{0} \\
\beta_{1}=\pi-\beta_{0}
\end{array}\right.
$$

Combining Equations (7) and (8), we get following result:

$$
\alpha_{1}+\beta_{1}=\pi-\gamma_{2}
$$

This proves that the planar quadrangle $I \boldsymbol{P}_{n, i}^{L} \boldsymbol{P}_{n, i} \boldsymbol{P}_{n-1, i}^{L}$ is inscribed in the circle circumscribed to the triangle $\boldsymbol{I} \boldsymbol{P}_{n, i}^{L} \boldsymbol{P}_{n-1, i}^{L}$. This remains true regardless the choice of the point $\boldsymbol{P}_{n, i}$ : the envelope of all possible $\boldsymbol{P}_{n, i}$ is on a circle. This property is preserved by inversion, and gives the proof of the second statement. We proceed in the same way for the third statement.

\section{Filling algorithm}

The filling algorithm with a circular mesh is valid if and only if the points $\boldsymbol{P}_{\boldsymbol{n}}$ and $\boldsymbol{P}_{\boldsymbol{n}}^{\prime}$ are identical. This discussion shows that this is equivalent to look for intersection of two circles belonging to a same sphere $S_{n}$. The algorithm proposes thus to determine the two circles and to compute their intersection and adapt the existing algorithm:

1. Choose arbitrarily the $\boldsymbol{P}_{\boldsymbol{i}}^{L}$, except $\boldsymbol{P}_{n-2}^{L}$;

2. Find $\boldsymbol{P}_{i}^{R}$ so that $\boldsymbol{P}_{\boldsymbol{i}}^{L}, \boldsymbol{P}_{\boldsymbol{i}+2}^{R}$ and $C_{i+1}$ belong to the same sphere.

3. Choose any point $\boldsymbol{P}_{\mathbf{0}}$ on the first circle.

4. Compute $\boldsymbol{P}_{n-2}^{L}$ by intersecting the two circles described in the previous paragraph.

5. Compute all the circles by propagation.

The additional step is not computationally intensive: the algorithm is almost identical to the one proposed in [5], but with more generality. It can be repeated ad libitum to the new hole generated in the middle of the new mesh. Note that the construction guarantees that there is one $\boldsymbol{P}_{n}^{\boldsymbol{R}}$ on the circle $C_{n}$ for any configuration of the $\boldsymbol{P}_{i}^{\boldsymbol{L}}$, but nothing guarantees that $\boldsymbol{P}_{n}^{\boldsymbol{R}}$ will be on the circular arc $V_{0} V_{n}$. Studying this question is beyond the scope of this paper, and we illustrate that irregular configurations can be achieved with the hole-filling algorithm in the next section.

\subsection{Implementation and numerical issues}

The framework proposed in this paper has been implemented within Grasshopper $^{\mathrm{TM}}$ and provide geometrical tools to generate the cyclidic nets and the associated subdivisions. Once the circular mesh is chosen, an infinity of frames can be chosen. All the underlying surfaces are $\mathrm{C}^{1}$, but some are visually more pleasant than others, as seen in Figure 14. To take this aesthetic aspect into account, a fairness-functional has been introduced to give the smoothest possible shape for a given circular mesh. The fairness function minimised here depends on the orientation of the frames, which can be measured with two angles made with a reference frame $\lambda$ and $\theta$. It is defined by:

$$
E_{\alpha}(\lambda, \theta)=\int\left(H^{2}-\alpha \kappa\right) d S
$$

- $\quad E_{0}=\int H^{2} d S$ is the Willmore energy; 
- $\quad E_{1}=\int\left(H^{2}-\kappa\right) d S$ is the conformally invariant Willmore energy;

- $E_{2}=\int\left(\kappa_{1}^{2}+\kappa_{2}^{2}\right) d S$ is a bending energy;

The case $\alpha=1$ has been extensively discussed in the domain of computer graphics: the energy obtained is invariant by inversions, which makes it particularly suited for a framework based on Möbius geometry [26]. It is commonly used as an aesthetic fairness measurement for shape modelling $[27,28]$. A discrete integration of $E_{\alpha}$ was implemented within the proposed framework, which minimizes automatically the bending energies of the cyclidic mesh by the means of a BFGS algorithm. In this paper, the value of $\alpha$ was set to 2. For the example of Figure 14, with 24 patches, the minimisation of the bending energy $E_{2}$ defined in equation (10) takes $150 \mathrm{~ms}$. Figure 14 shows two surfaces: one with a random choice of frame, and the other with an optimised value of the bending energy. The second surface is visually very regular and validates the choice of fairness functional made in this paper.

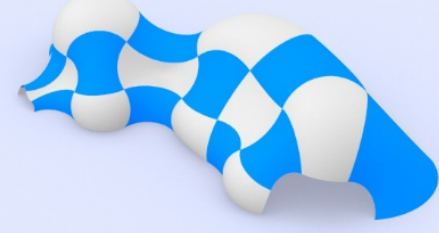

(a) $\mathrm{E}_{2}=112.0$

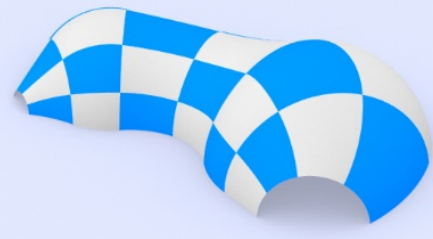

(b) $E_{2}=29.9$

Figure 14: Two cyclidic nets supported on the same circular mesh, before and after optimization of the bending energy

The same fairness functional is used for the solution of the hole-filling problem. The hole-filling algorithm yields surfaces of varying smoothness, depending on the boundaries. Figure 15 shows some solutions of the hole-filling problem: the location of the singularity remains almost unchanged while several smooth configurations can be obtained with the strategy proposed in this paper. However, the smoothest configuration (Figure 15, middle) corresponds to the case where the hole is shrunk towards a regular hexagon. It is also the minimum of the chosen bending energy, which validates again the choice for $E_{2}$ as a fairness function. Notice finally that the resulting shape is non-symmetrical: this is made possible by the hole filling strategy used in this paper.
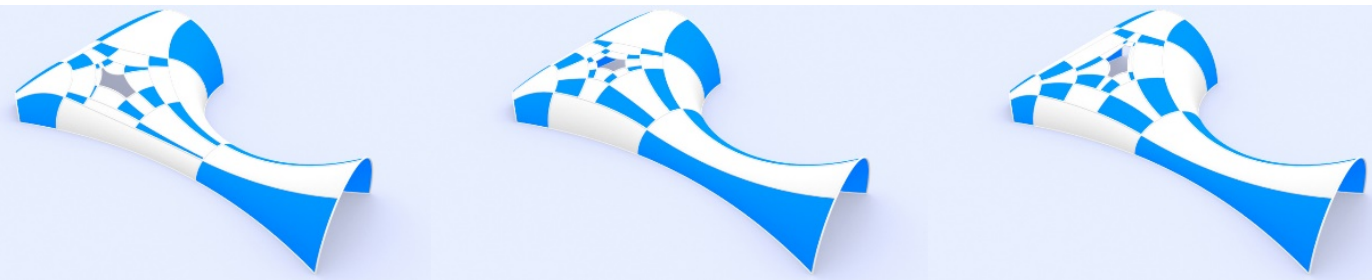

Figure 15: Several solutions obtained with the hole-filling problem for an irregular hole. Values of $E_{2}$, from left to right: $38.7,36.5,38.2$

\section{MODELLING WITH GENERALISED CYCLIDIC NETS}

\subsection{Shape generation framework}

The study of cyclidic nets demonstrates that a circular mesh and a frame can generate a collection of surface parameterised by their lines of curvature. This 
collection of surfaces can then be easily meshed with conical or circular PQ-Meshes or planar hexagonal meshes and can be smooth or creased. Unlike previous applications of cyclidic meshes in architecture, it is suggested by the authors that the shapes can be described with large cyclidic patches, the subdivision of the patches being kept as a design parameter serving functions other than form, for example mechanical performance. The result is a framework for shape modelling tailored for architectural constraints.

This framework is presented in Figure 16. Starting from a coarse circular mesh, it is possible to generate a generalised cyclidic net. The designer is free to add normal discontinuity with our method at this step. One can then choose a panelisation strategy, depending on constructional constraints or aesthetics. The most common design strategies for structural and envelope layout yield interesting geometrical properties without post-rationalisation. This method is thus a bottom-up approach, allowing designers to check different construction solutions for the same shape. Figure 17 illustrates two technological alternatives generated with our framework, and Figure 2 represents a third alternative on a different shape.

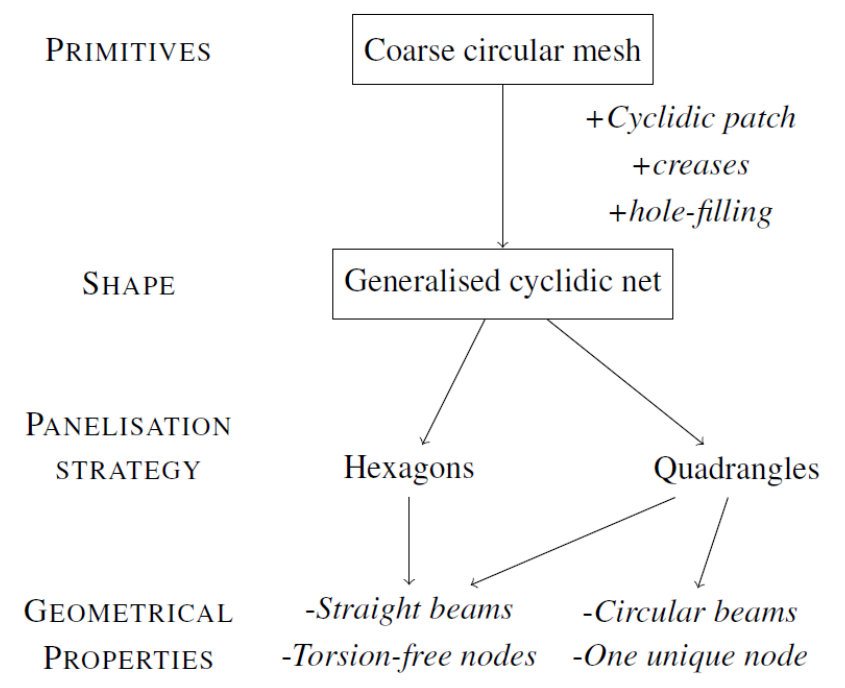

Figure 16: Framework for shape generation with generalised cyclidic nets

A brief comparison with NURBS is proposed in Table 1. A parallel can be found between the control polygon of NURBS and the circular mesh of cyclidic nets. Unlike control points in NURBS modelling, the vertices of Cyclidic Nets are all on the modelled surface. The surface resulting from cyclidic nets are only $\mathrm{C}^{1}$, which is a drawback in many industries, but is not a very serious issue in architectural design. Indeed, the final shape is very often built with flat or developable panels, which makes the built envelope at most a $C^{1}$ surface. Finally, it is noticed that NURBS have some limitations when complex topologies are concerned, a popular alternative is the use of subdivision surfaces, mainly based on the Catmull-Clark subdivision scheme [29].

Table 1: Comparison of the NURBS and Generalised Cyclidic Net frameworks

\begin{tabular}{|l|l|l|}
\hline Property & NURBS & $\begin{array}{l}\text { Generalised cyclidic } \\
\text { nets }\end{array}$ \\
\hline Base shape & Control Polygon. & $\begin{array}{l}\text { Circular Quad Mesh + one } \\
\text { frame }\end{array}$ \\
\hline
\end{tabular}




\begin{tabular}{|l|l|l|}
\hline Interpolation & Bernstein polynomial & Cyclidic patch \\
\hline Surface regularity & From $\mathrm{C}^{0}$ to $\mathrm{C}^{\infty}$ & $\mathrm{C}^{0}$ or $\mathrm{C}^{1}$ \\
\hline $\begin{array}{l}\text { Isoparametric lines } \\
\text { properties }\end{array}$ & None & Curvature lines \\
\hline Complex topologies & Subdivision surfaces & Hole-filling strategy \\
\hline
\end{tabular}

\subsection{Primitives for circular meshes}

The framework proposed here requires thus circular meshes as input. Some shapes give trivial conical or circular meshes. Among them, one can find surfaces of revolution, moulding surfaces or Monge surfaces [7]. It is possible to apply Möbius transforms to those shapes to enrich the formal vocabulary: some case studies are presented in this paper. Composition of these shapes is also possible in the manner of what has been proposed for scale-trans surfaces [1].

This framework can be combined with the optimisation methods described in [30, 31], with an optimisation of quad meshes towards circular meshes. The combination of those methods has several advantages. First of all, since cyclidic patches are smooth surfaces, only a few of them are required to describe a given shape: this decreases the size of the problems to be solved by optimisation and makes shape exploration easier. Secondly, they can be easily re-meshed with no computational optimisation.
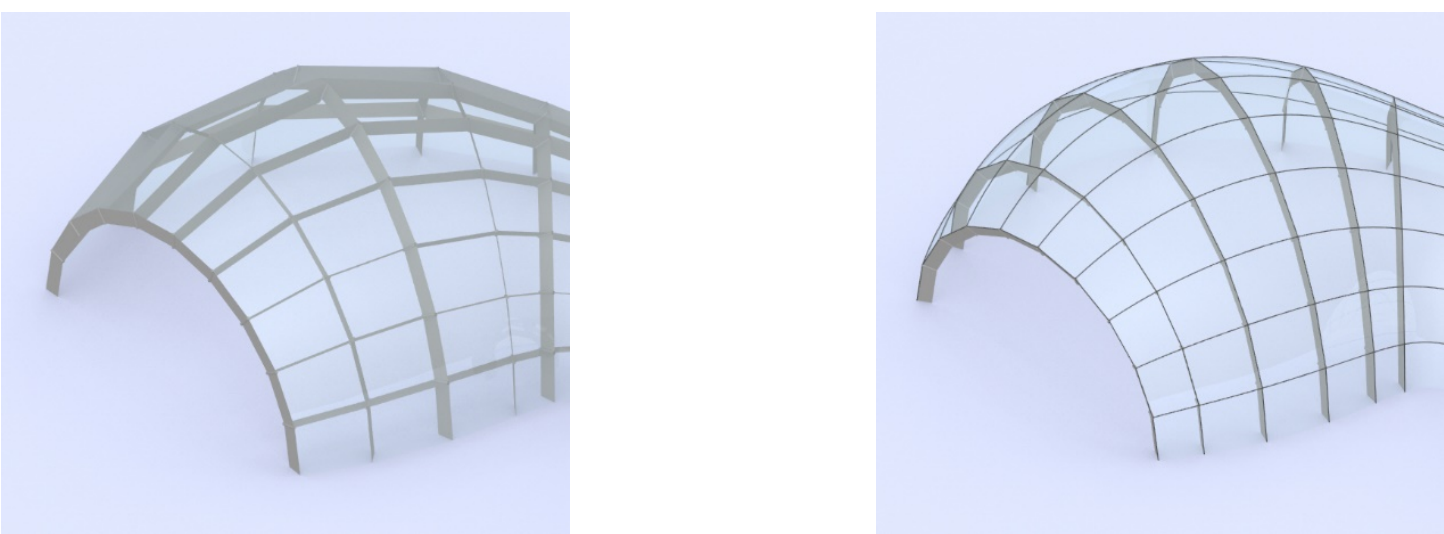

Figure 17: Some technological possibilities offered by cyclidic nets. Left: flat panels, straight members with torsion-free nodes. Right: D-strips, straight members with torsion-free nodes.

The hole-filling strategy introduced in this paper allows the modelling of complex topologies, such as the shape shown in Figure 18. In this image, the thicker arches correspond to boundaries of cyclidic patches. The grid structure is covered with quadrangular panels, except two hexagons. 

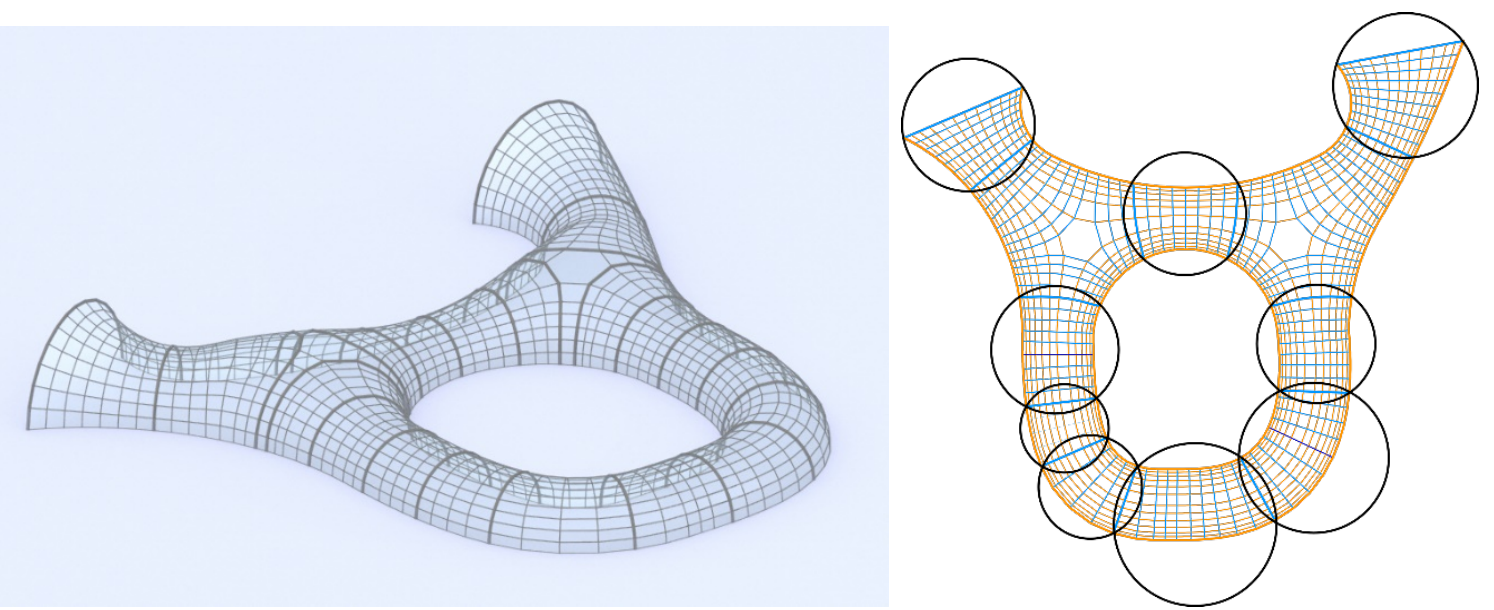

Figure 18: A geometry with a complex topology modelled with generalised cyclidic nets. Left: rendering of the shape. Right: the circles supporting the cyclidic patches.

All the circles supporting the patches lie on the $X Y$ plane, with the exception of the patches used for the hole-filling strategy. Figure 18 shows the circles used for the generation of this shape. The holes are non-symmetrical hexagons. The holes boundaries fulfil the condition described in Equation (6). The control of the shape can be done by moving the circles in the (XY) plane.

This shape vocabulary can easily be extended with the use of inversion or transformations by parallelism, also known as Combescure transformations [15]. The latter transformations have been employed in [7], where properties of node repetition in surfaces revolutions have been extended to moulding surfaces.

\subsection{Shape modelling with inversions}

Möbius transforms applied on circular meshes offer a rich variety of shapes to support cyclidic nets, as they preserve circles. They considerably enrich the design possibilities of conventional geometries. An example is illustrated in Figure 19 and 20: a simple surface of revolution is inverted to give a less obvious 'peanut-shaped' geometry. The inversion of surfaces of revolution allows to model very efficiently shapes recalling existing projects, like the forum of the Soliday's festival [32].

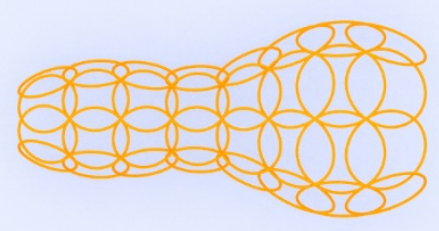

(a) Surface of revolution: circumcircles

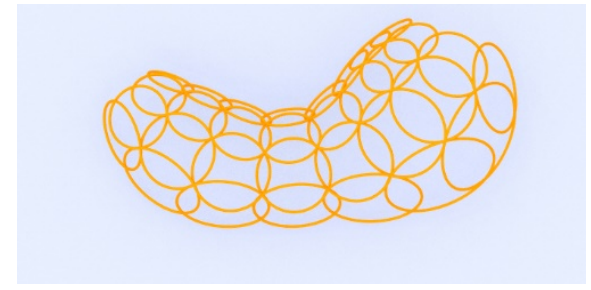

(c) Inversion: circumcircles

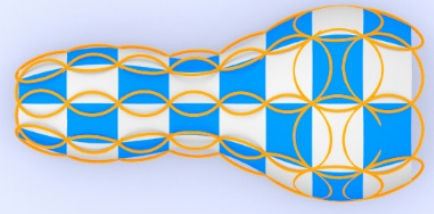

(b) Surface of revolution as a cyclidic net

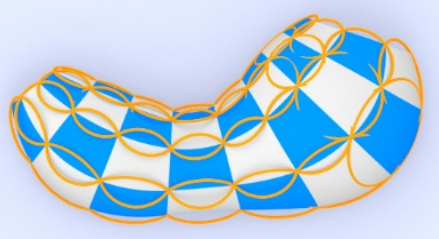

(d) Inversion: cyclidic net 
Figure 19: A surface of revolution and an inversion to a 'peanut-shaped' geometry The computation of the Möbius transform is based on Equation (1). It requires no optimisation or matrix manipulation and can therefore be done as quickly as other simple transformations, like translations. The cyclidic net lying on the circular mesh is also generated in real-time, since it only consists in the propagation of a frame along the net. The shape can then be subdivided in a uniform pattern.
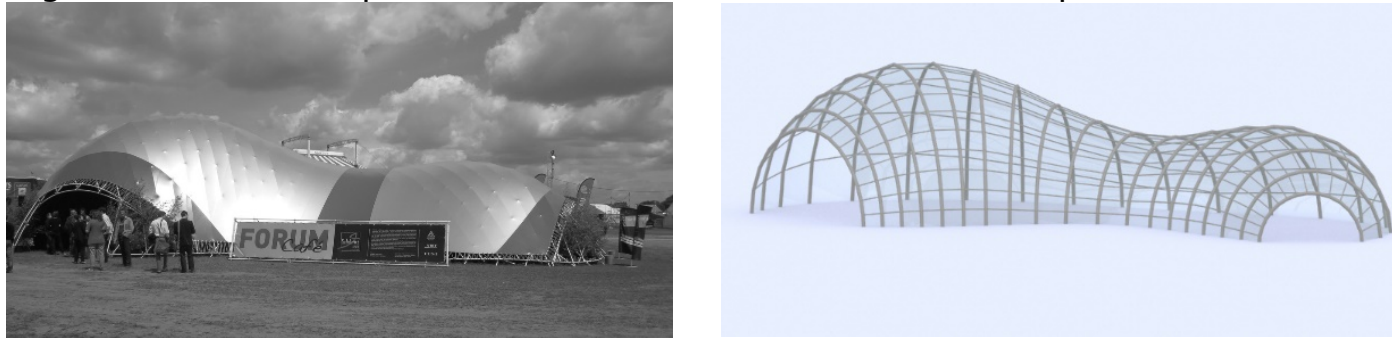

Figure 20: The 'peanut' gridshell of the Solidays forum: built project and interpretation with the inversion of a surface of revolution.

Möbius transforms can be applied on more complex shapes than surfaces of revolution, for example on Monge's surfaces or moulding surfaces [7]. Figure 21 shows some inversions of the mesh displayed in Figure 17, the reference geometry is at the bottom right of Figure 21. Although the Möbius transform does locally preserve the shapes, the overall aspect of the models varies substantially. All the shapes displayed in this image can be covered with circular meshes and be parameterised by cyclidic nets. To better understand the deformation of surfaces by spherical inversions, it is convenient to consider the degrees of freedom that they offer. A spherical inversion is defined by four parameters introduced in Equation (1): the three coordinates of the centre of inversion $\mathbf{C}$ and the ratio $k$. One can also consider the steering of form through the direct manipulation of points on a surface. There is a one-parameter family of spherical inversions with an arbitrary image of three points in 3D. In general, one can however not find an inversion through the prescription of the image of four points. Möbius transformations offer more control than usual transformations, like shearing deformations.

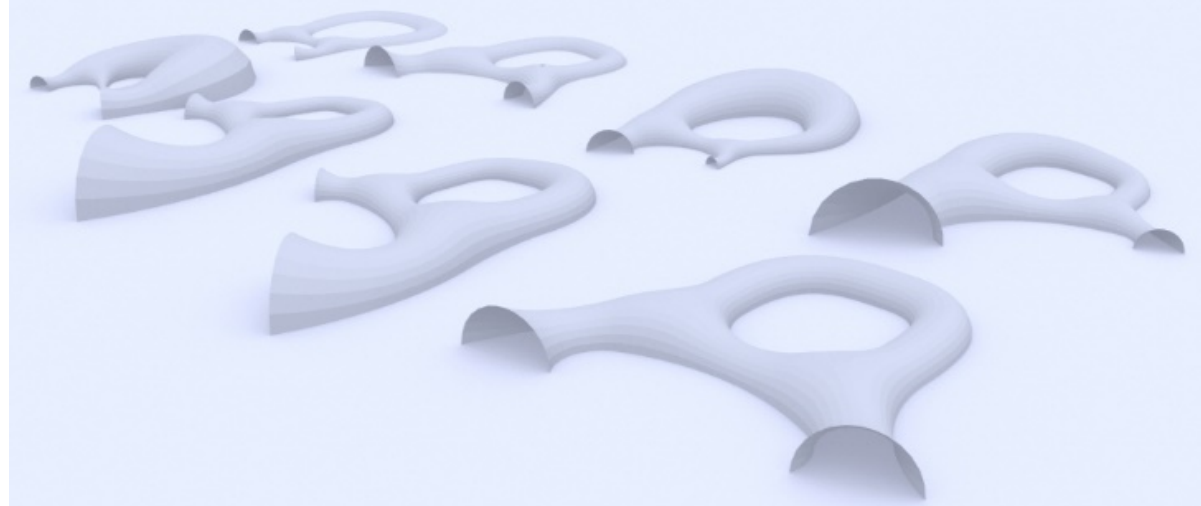

Figure 21: A family of shapes produced by inversions of the same geometry.

\subsection{Application of curved creases}

Generalised cyclidic nets offer the possibility to create non-smooth surfaces with a straight forward folding strategy. An example of this strategy is proposed in Figure 22: the same base circular mesh is used for three designs of a tribune cover but 
different values have been chosen for the folding angle $\theta$. All the surfaces are regularized with the bending energy introduced in Equation (10). All solutions are visually regular, which further demonstrates that such energy is appropriate, even for $\mathrm{C}^{0}$ surfaces. Although the overall geometry is preserved, the visual aspects of the three solutions varies significantly.

The three solutions can lead architects and engineers to choose different technological solutions. The smooth surface is more suited for a grid structure. The two other solutions increase significantly the local curvature. The variant with $\theta=225^{\circ}$ recalls tensile structure, which are known for their efficient use of material. The variant with $\theta=135^{\circ}$ works with compression-dominant forces. It recalls some of Nicolas Esquillan's shells, where creases were used to increase the buckling capacity of thin shells.

(c) $\theta=135^{\circ}$

(a) $\theta=180^{\circ}$

(b) $\theta=225^{\circ}$

Figure 22: Three different generalised cyclidic nets supported on the same circular mesh. All shapes can easily be covered with planar facets and clean supporting beam layout.

These considerations on the mechanical behaviour of corrugated shells are confirmed here with finite element analysis. Generalised cyclidic nets offer the possibility to generate efficient structures as illustrated in $[23,24]$, but a detailed structural analysis should be made systematically to confirm the relevance of doubly curved creases.

\section{CONCLUSION}

The fabrication of free-form structures remains a challenge for designers. Engineers and architects need interactive tools to assess the constructability of their designs. Both NURBS modelling and mesh optimisation strategy have taken an increasing importance in complex structures, but they show some limitations in that regard. In this paper, the authors have introduced a method spanning between these two solutions that offers various technological alternatives for fabrication. Generalised Cyclidic Meshes use a base circular mesh and generate complex shapes as a collection of NURBS parameterised by their lines of curvature, yielding automatically geometrically optimal structural layouts. This framework for fabrication-aware design also introduces a systematic way to generate constructible creases in free-form architecture, which offers new design possibilities. It creates new shapes thanks to inversion, and the potential offered by curved crease in structural optimisation seems very promising.

The potential of the method seems promising, although developments are necessary to make it a usable tool for the designers. For example, the implementation of Boolean operations is a necessity for practical modelling. This specific issue should be studied further. Future work also includes the development of a user-friendly environment for the design of creased shells, exploration of the design space offered by creased shells and automatic methods for the generation of circular meshes. The extension of these findings for other patterns, like the tri-hex pattern, should also be 
studied. Innovative methods of structural optimisation combining form-finding and creases can also arise from this work.

\section{REFERENCES}

1. Motro R., Structural Morphology, Journal of the International Association for Shells and Spatial Structures, Special Issue 7 papers, 1993, 34.

2. Bagneris M. et al., Structural Morphology Issues in Conceptual Design of Double Curved Systems, International Journal of Space Structures, 2008, 23(2), 79-87.

3. Glymph, J. et al. A parametric strategy for free-form glass structures using quadrilateral planar facets. Automation in Construction, 2004; 13(2), 187202.

4. Mesnil R. et al., Möbius Geometry and Cyclidic Nets: A Framework for Complex Shape Generation. In Proceedings of the IASS: Future Visions, Amsterdam, 2015.

5. Krasauskas R., Circular Meshes, cyclidic splines and geometric algebra. In Algebraic geometry in the sciences, Oslo, 2011.

6. Liu, Y. et al., Geometric Modeling with Conical Meshes and Developable Surfaces. ACM Transactions on Graphics, 2006; 25(3): 681-689.

7. Mesnil, R., Douthe, C., Baverel, O., Léger, B., \& Caron, J.-F. Isogonal moulding surfaces: a family of shapes for high node congruence in free-form structures. Automation in Construction, 2015; 59, 38-47.

8. Wallner, J. \& Pottmann, H. Geometric computing for freeform architecture, Journal of Mathematics in Industry, 2011, 1(4).

9. Pottmann, H., \& Wallner, J. The focal geometry of circular and conical meshes. Advances in Computational Mathematics, 2006; 29, 249-268.

10. Bobenko, A., Matthes, D., \& Suris, Y. B. Discrete and smooth orthogonal systems: $C^{\infty}$-approximation. International Mathematics Research Notices, $2003 ; 45,2415-2459$.

11. Wang, W., Liu, Y., et al. Hexagonal Meshes with Planar Faces. Dept. of CS, HKU, Tech. Rep. 2008.

12. Douthe, C., \& Baverel, O. (2009). Design of nexorades or reciprocal frame systems with the dynamic relaxation method. Computers \& Structures, 2009; 87(21-22), 1296-1307.

13. Bobenko, A. Surfaces from Circles. In Discrete Differential Geometry, 2008; 38, 3-35. Springer. 
14. Adam P., Sur les surfaces isothermiques à ligne de courbure planes dans un système ou deux systems. Annales scientifiques de I'E.N.S. $1893 ; 10$ :319358.

15. Pottmann, H., Liu, Y., et al. Geometry of multi-layer freeform structures for architecture. ACM Transactions on Graphics, 2007; 26(3), 65.

16. Mesnil R. et al. Linear buckling of quadrangular and kagome gridshells: a comparative assessment, Engineering Structures, 2017; 132(1), 337-348.

17. Garnier, L., Foufou, S., \& Neveu, M. Conversion d'un carreau de Bézier rationnel biquadratique en un carreau de cyclide de Dupin quartique. TSI. Technique et Science Informatiques, 2006; 25(6), 709-734.

18. Bobenko, A., \& Huhnen-Venedey, E. Curvature line parametrized surfaces and orthogonal coordinate systems: discretization with Dupin cyclides. Geometriae Dedicata, 2012; 159(1), 207-237.

19. Bo, P. et al.. Surface fitting with cyclide splines, Bo et al., Computer Aided Geometric Design, 2016, 43, 2-15.

20. McLean, D. A method of Generating Surfaces as a Composite of Cyclide Patches. The Computer Journal, 1985; 28(4), 433-438.

21. Dutta, D., Martin, R. R., \& Pratt, M. J. Cyclides in surface and solid modeling. IEEE Computer Graphics and Applications, 1993; 13(1), 53-59.

22. Lebée A., From Folds to Structure: a Review, International Journal of Space Structures, 2015, 30(2),55-74.

23. Motro R. \& Maurin B., Bernard Lafaille and Nicolas Esquillan: Two French Pioneers. In IASS-IABSE Symposium:Taller, Longer, Lighter , 2011.

24. Mesnil R. et al. Structural Explorations of a fabrication-aware design space with marionette meshes. In Proceedings of the IASS Annual Symposium 2016 - Spatial Structures in the $21^{\text {st }}$ Century, 2016.

25. Liu, Y., \& Wang, W. On Vertex Offsets of Polyhedral Surfaces. In Proceeding of Advances in Architectural Geometry, 2008, (pp. 61-64).

26. Bobenko, A., \& Schröder, P. Discrete Willmore Flow. In Proceedings of the third Eurographics symposium on Geometry processing. Eurographics Association, 2005; p. 101

27. Séquin, C. H. CAD Tools for Aesthetic Engineering. Computer-Aided Design, 2005; 37(7), 737-750.

28. Joshi, P., \& Séquin, C. H. Energy Minimizers for Curvature-Based Surface Functionals. Computer-Aided Design and Applications, 2007; 4(5), 607-617.

29. Catmull, E., \& Clark, J. Recursively generated B-spline surfaces on arbitrary topological meshes. Computer-Aided Design, 1978; 10(6), 350-355. 
30. Deng, B., et al.. Interactive design exploration for constrained meshes. Computer-Aided Design, 2015; 61, 13-23.

31. Yang, Y., Yang, Y.-J., Pottmann, H., \& Mitra, N. J. Shape Space Exploration of Constrained Meshes. ACM Transactions on Graphics, 2011; 30, 124.

32. Tayeb, F., Caron, J.-F., Baverel, O., \& Du Peloux, L. Stability and robustness of a $300 \mathrm{~m}^{2}$ composite gridshell structure. Construction and Building Materials, 2013; 49, 926-938. 